\title{
Clinical feasibility and application value of computer virtual reduction combined with $3 D$ printing technique in complex acetabular fractures
}

\author{
LEI WAN, XIAOGUANG ZHANG, SHAOAN ZHANG, KUI LI, PENGKE CAO, \\ JUNMING LI and GUANGLIANG WU
}

Department of Orthopedics, The Second Affiliated Hospital of Luohe Medical College, Luohe, Henan 462300, P.R. China

Received July 16, 2018; Accepted January 22, 2019

DOI: 10.3892/etm.2019.7344

\begin{abstract}
This study investigated the clinical feasibility and application value of computer virtual reduction combined with three-dimensional (3D) printing technique in patients with complex acetabular fracture. Ninety-six patients diagnosed with complex acetabular fracture in the Orthopedics Department in The Second Affiliated Hospital of Luohe Medical College from January 2016 to June 2017 were selected and randomly divided into the routine operation group $(n=48)$ and the 3D model group $(n=48)$ according to the admission number of the patients. In the 3D model group, computed tomography (CT) scan was performed preoperatively, and the model was made using the virtual technique and 3D printing technique. The surgical scheme was designed according to the model. Patients in the routine operation group were diagnosed with the conventional CT scan without using the computer virtual technique and 3D printing technique. During operation, the operation time, amount of intraoperative bleeding and times of intraoperative fluoroscopy were recorded in both groups. After operation, the incidence rate of such complications as inflammatory response, iatrogenic neurological symptoms and loss of reduction were recorded in both groups. Moreover, the reduction quality of acetabular fracture was evaluated according to the Matta imaging score at 3 days after operation, and the hip joint function was evaluated based on the Hariss score at 6 months after operation. In the 3D model group, the operation time was significantly shorter than that in the control group, the amount of intraoperative bleeding and times of intraoperative fluoroscopy were significantly less than those in the routine operation group, and the incidence rate of postoperative complications was obviously lower than
\end{abstract}

Correspondence to: Dr Guangliang Wu, Department of Orthopedics, The Second Affiliated Hospital of Luohe Medical College, 463 Haihe Road, Yancheng, Luohe, Henan 462300, P.R. China

E-mail:w2q5mk@163.com

Key words: acetabular fracture, computer virtual, 3D printing technique that in the routine operation group $(\mathrm{P}<0.05)$. In conclusion, computer virtual reduction combined with the $3 \mathrm{D}$ printing technique can significantly reduce the operation time, amount of intraoperative bleeding, times of intraoperative fluoroscopy and incidence rate of postoperative complications without adverse effects on the reduction quality of acetabular fracture and hip joint function of patients, which has a higher clinical application value and greater social significance.

\section{Introduction}

The acetabulum is an important component of the human hip joint, as well as the largest movable joint and weight-bearing joint in the human body, which can be easily damaged. The acetabulum is characterized by well-developed surrounding muscles and deep joint position, and it is surrounded by complex nerves and blood vessels. The acetabular fracture is mostly caused by central dislocation of hip joint, hip fracture or pubic fracture involving the acetabulum and so on (1). The acetabular fracture is the intra-articular fracture caused by high-energy injury, accounting for approximately 3\% of systemic fractures, which can cause damage to articular surface in severe cases (2). There are complex and diverse acetabular fractures. At present, major therapeutic methods in the western medicine for acetabular fracture include conservative treatment and operative treatment. However, acetabular fracture is often accompanied with obvious displacement of more than $3 \mathrm{~mm}$, so it is hard for conservative treatment to reduce the femoral head and acetabulum, and it can also lead to femoral head necrosis, ankylosis and other hip dysfunctions (3). Since Judet et al (4) adopted operation in the treatment of acetabular fracture for the first time, operative treatment for acetabular fracture has been gradually matured and become a mainstream therapeutic method in the Orthopedics Department in The Second Affiliated Hospital of Luohe Medical College (Luohe, China). Compared with conservative treatment, operative treatment is characterized by a short treatment cycle, early weight-bearing time and decreased incidence rate of complications (5). However, due to the high difficulty of anatomy of acetabulum joint, difficulty in intraoperative exposure, small operative and visual space and other adverse factors, how to avoid the damage to the nerves, blood vessels and organs during operation has 
become the key to successful operation. The treatment of acetabular fracture aims to restore the biomechanical property of pelvis and acetabulum through reconstructing the articular surface and restoring the anatomical structure (6). However, there are certain limitations in the conventional X-ray and computed tomography (CT) scan in the operative plan, often leading to certain defects in the operative plan. Improper operative plan may result in large exposure area, long operation time, large amount of bleeding, ectopic ossification and other complications (7). Therefore, the precise, individualized and optimized preoperative design is necessary for the operation.

In recent years, with the in-depth application of computer-aided technology and three-dimensional (3D) printing technique in the field of orthopedics, it has been gradually transformed from virtual simulation to realistic simulation. Clinicians, through the 3D printing model, can perform minimally-invasive reduction of surgical site, optimize the surgical approach and realize the precise design and simulation operation, thereby reducing the operative complications and ensuring a successful operation. In this study, therefore, the clinical feasibility and application value of computer virtual reduction combined with 3D printing technique in the patients with complex acetabular fracture were investigated with conventional operation as the control.

\section{Patients and methods}

General data. A total of 96 patients diagnosed with complex acetabular fracture via complete pelvic CT scan in the Orthopedics Department in The Second Affiliated Hospital of Luohe Medical College from January 2016 to June 2017 were selected and randomly divided into the routine operation group $(n=48)$ and the 3D model group $(n=48)$ according to the admission number of the patients. This study was approved by the Ethics Committee of The Second Affiliated Hospital of Luohe Medical College, and all patients voluntarily signed the informed consent.

Inclusion criteria. i) Patients diagnosed with complex hip fracture and fracture of acetabular posterior wall via CT scan, including the T-shaped type, anterior + transverse type, both-column type, posterior wall type, posterior column + posterior wall type and transverse + posterior wall type, ii) patients aged 18-64 years, iii) patients with duration from injury to operation of less than 2 weeks, and iv) patients who voluntarily signed the informed consent.

Exclusion criteria. i) Patients diagnosed with a single fracture via CT scan, including posterior wall fracture, posterior column fracture, anterior wall fracture, anterior column fracture and transverse fracture, ii) patients with delayed acetabular fracture, iii) patients aged $<18$ years, iv) patients complicated with vascular injury, v) patients with poor mental status or confusion of consciousness, vi) patients with severe cardiovascular or cerebrovascular diseases or hepatorenal syndrome, or vii) patients with immune system diseases.

\section{Methods}

Preoperative treatment. After admission, routine examinations, such as biochemical and cardiopulmonary function examinations, were performed for patients. Patients accompanied with posterior dislocation of hip joint were treated with manual reduction of acetabular dislocation and skeletal traction of $6-8 \mathrm{~kg}$ on the femoral condyle on the affected side. The pelvic orthotopic, obturator oblique and Iliac oblique X-ray films of patients were examined, and CT plain scan and $3 \mathrm{D}$ reconstruction were performed. Patients complicated with severe craniocerebral and chest and abdominal injury should be treated first, followed by operative treatment after the patient's condition became stable. Certain anti-coagulant and anti-infection treatments were given before the operation.

On the basis of the above examination, the patients in the 3D model group were required to perform a $1.0 \mathrm{~mm}$ CT scan (Aquilion 64; Toshiba, Tokyo, Japan) on the thin pelvic layer, and the data were kept in the DICOM format. The editing function of Mimisc14.0 (Materialise, Belgium) was used to separate the pelvis from the femur bone and remove the femur image. The acetabular and fracture blocks were used to increase the function of the area, and the acetabular body and the fracture blocks with clinical significance were completely divided. In the 3D modeling window, the displacement and rotation function was used to restore each fracture block. The data before and after reduction were saved as STL format and sent to MakerBot Replicator 2 printer, and the 3D physical fracture model was printed as 1:1. According to the 3D physical fracture model before the reduction, the fracture classification, fracture displacement degree, the deformation direction, rotation direction and angle of each fracture block were further defined. The whole process of the operation was simulated, including the selection of the incision, the sequence of reduction, the placement of the reduction clamp, the rotation direction, the specific placement of the steel plate and screw, the angle, the length, and the degree of pre-bending, so as to make it as reasonable as possible to achieve effective strong fixation. The effect of reduction was evaluated according to the $3 \mathrm{D}$ physical fracture model after reduction.

Surgical approach. According to the acetabular fracture of patients in the 3D physical model, the supine position or lateral floating position was selected. Kocher-Langenbeck approach was selected for the posterior column fracture accompanied with posterior wall fracture and transverse fracture accompanied with posterior wall fracture, ilioinguinal approach was selected for the anterior fracture accompanied with transverse fracture, and anterior-posterior combined approach was selected for the both-column fracture. The approach for T-shaped fracture was designed based on the patient's fracture morphology.

Operation methods. In the 3D model group, after tracheal intubation and general anesthesia, the hip on the affected side of patients was underlaid for 45 degrees in a hemi-lateral position, and the internal surface and anterior column of the pelvis were exposed via the ilioinguinal approach. The hip on the affected side was pushed forward until 90 degrees in a lateral position, and the internal surface, anterior and posterior columns and posterior wall of the pelvis were exposed via the Kocher-Langenbeck approach. Then the pre-bent steel plate was inserted into the fracture site and fixed with the pre-designed screws. The operation was completed after the intraoperative X-ray fluoroscopy displayed good reduction and fixation of the fracture. In the control group, the conventional 
Table I. Comparison of general data of patients in two groups.

\begin{tabular}{|c|c|c|c|c|c|c|}
\hline \multirow[b]{2}{*}{ Groups } & \multicolumn{2}{|c|}{ Sex } & \multicolumn{2}{|c|}{ Age (years) } & \multicolumn{2}{|c|}{$\begin{array}{l}\text { Time from injury } \\
\text { to operation (days) }\end{array}$} \\
\hline & Male & Female & Mean & Range & Mean & Range \\
\hline 3D model group & 34 & 14 & $43.44 \pm 4.53$ & $20-61$ & $10.12 \pm 1.41$ & $4-14$ \\
\hline Routine operation group & 32 & 16 & $41.88 \pm 4.97$ & $19-62$ & $10.41 \pm 1.06$ & $5-14$ \\
\hline$\chi^{2} /$ t-test & \multicolumn{2}{|c|}{0.19} & 1.61 & \multicolumn{3}{|c|}{1.29} \\
\hline P-value & \multicolumn{2}{|c|}{0.65} & 0.11 & \multicolumn{3}{|c|}{0.19} \\
\hline
\end{tabular}

reduction and fixation of acetabular fracture were performed after X-ray fluoroscopy and CT scan.

Postoperative treatment. The broad-spectrum antibiotics were applied for $24 \mathrm{~h}$ after operation, and the low-molecular-weight heparin was subcutaneously injected for 2 weeks. The muscle contraction function on the affected side was exercised 1 day after operation. The knees and hip joints on the affected side were exercised 3 days after operation, and they could be moved actively at 2 weeks. Patients could exercise out of bed protectively at 4 weeks. Weight-bearing activity was strictly forbidden within 3 months. After 3 months, patients could gradually take full weight-bearing activity according to their recovery status.

Evaluation indexes. Operation time (min): The time from skin incision to suture in both groups was recorded.

Amount of intraoperative bleeding (ml) (8): Amount of intraoperative bleeding $=$ (intraoperative amount of liquid absorbed by the gauze + amount of liquid sucked by the autologous blood transfusion equipment + amount of liquid sucked on the wound surface) - amount of washing liquid.

Times of intraoperative fluoroscopy (times): Fluoroscopy was performed during operation to effectively observe the reduction of fracture or internal fixation status of patients.

Incidence rate of postoperative complications (\%) (9): After operation, the incidence rate of such complications as inflammatory response, iatrogenic neurological symptoms, loss of reduction, ectopic ossification and traumatic arthritis were recorded in both groups.

Score of reduction quality of acetabular fracture (points): $\mathrm{X}$-ray examination was performed for review 3 days after operation, and the reduction of fracture was evaluated according to the Matta imaging scoring criteria (10). Evaluation criteria: Excellent, residual displacement of fracture $<1 \mathrm{~mm}$; good, residual displacement of fracture $=2-3 \mathrm{~mm}$; and poor, residual displacement of fracture $>3 \mathrm{~mm}$.

Hip joint function score (points): The hip joint function was evaluated based on the Harris score (11) at 6 months after operation. Evaluation criteria: A total of 100 points (excellent, $>90$ points; good, $80-89$ points; fine, $70-79$ points; and poor, $<70$ points).

Statistical analysis. Statistical Product and Service Solutions (SPSS) 17.0 (SPSS, Inc., Chicago, IL, USA) was used for statistical analysis. Measurement data were expressed as mean \pm standard deviation (mean $\pm \mathrm{SD}$ ), and t-test was used. Enumeration data were expressed as percentage, and
Chi-square test was adopted. $\mathrm{P}<0.05$ suggested that the difference was statistically significant.

\section{Results}

Comparisons of general data and acetabular fractures of patients in two groups. There was no statistically significant difference between the two groups in sex, average age, average injury time and acetabular fracture classification $(\mathrm{P}>0.05)$ (Tables I and II).

Comparison of operation time, amount of intraoperative bleeding and times of intraoperative fluoroscopy between the two groups. There were statistically significant differences in the comparison of operation time and times of intraoperative fluoroscopy between the two groups of patients $(\mathrm{P}<0.05)$. The amount of intraoperative bleeding in the 3D model group was significantly less than that in the routine operation group, showing a statistically significant difference $(\mathrm{P}<0.05)$. The number of intraoperative fluoroscopy was compared between the two groups, and the difference was statistically significant $(\mathrm{P}<0.05)$ (Table III).

Comparison of incidence rate of postoperative complications between the two groups. There were 1 case of inflammatory response, 1 case of iatrogenic neuropathy, 1 case of heterotopic ossification and 2 cases of traumatic arthritis in the 3D model group. In the routine operation group, there were 6 cases of inflammatory reaction, 5 cases of iatrogenic nerve symptoms, 3 cases of heterotopic ossification and 4 cases of traumatic arthritis. The number of postoperative complications was compared between the two groups, and the difference was statistically significant $(\mathrm{P}<0.05)$ (Table IV).

Comparison of excellent-good rates of reduction quality of acetabular fracture and hip joint function after operation between the two groups. There were no statistically significant differences in the comparison of excellent-good rates of reduction quality of acetabular fracture at 3 days after operation and hip joint function at 6 months after operation between the two groups of patients $(\mathrm{P}>0.05)$ (Table V).

Acetabular fracture morphology before and after operation. The acetabular fracture morphology before and after operation is shown in Fig. 1. The patient suffered from severe right acetabular fracture (Fig. 1A). The 3D physical model 
Table II. Comparison of acetabular fractures of patients in two groups.

\begin{tabular}{lcccc}
\hline Characteristics & $\begin{array}{c}\text { 3D model } \\
\text { group }\end{array}$ & $\begin{array}{c}\text { Routine operation } \\
\text { group }\end{array}$ & $\chi^{2}$ test & P-value \\
\hline Acetabular fracture classification & & & & \\
T-shaped fracture & 7 & 8 & 0.07 & 0.77 \\
Posterior column fracture accompanied with posterior wall fracture & 12 & 11 & 0.06 & 0.81 \\
Both-column fracture & 8 & 9 & 0.07 & 0.79 \\
Transverse fracture accompanied with posterior wall fracture & 11 & 12 & 0.06 & 0.81 \\
Anterior fracture accompanied with transverse fracture & 7 & 6 & 0.08 & 0.76 \\
Acetabular marginal fracture & 2 & 1 & 0.34 & 0.55 \\
Compression fracture of articular surface of acetabulum & 2 & 1 & 0.34 & 0.55 \\
Associated injury & & & & \\
Limb fracture & 26 & 25 & 0.04 & 0.83 \\
Chest and abdominal injury & 13 & 14 & 0.05 & 0.82 \\
Craniocerebral injury & 8 & 9 & 0.07 & 0.78 \\
\hline
\end{tabular}

Table III. Comparison of operation time, amount of intraoperative bleeding and times of intraoperative fluoroscopy between the two groups (mean $\pm \mathrm{SD})$.

\begin{tabular}{|c|c|c|c|c|}
\hline Groups & $\mathrm{n}$ & $\begin{array}{l}\text { Operation time } \\
(\min )\end{array}$ & $\begin{array}{c}\text { Amount of intraoperative } \\
\text { bleeding }(\mathrm{ml})\end{array}$ & $\begin{array}{c}\text { Times of intraoperative } \\
\text { fluoroscopy }\end{array}$ \\
\hline 3D model group & 48 & $210.8 \pm 54.5$ & $1,147.2 \pm 235.4$ & $6.8 \pm 1.6$ \\
\hline Routine operation group & 48 & $296.4 \pm 66.2$ & $1,832.5 \pm 268.1$ & $12.4 \pm 2.1$ \\
\hline t-test & & 6.92 & 13.31 & 14.7 \\
\hline P-value & & $<0.001$ & $<0.001$ & $<0.001$ \\
\hline
\end{tabular}

Table IV. Comparison of the number of patients with postoperative complications between the two groups.

\begin{tabular}{|c|c|c|c|c|c|}
\hline Groups & $\mathrm{n}$ & $\begin{array}{l}\text { Inflammatory } \\
\text { response }\end{array}$ & $\begin{array}{l}\text { Iatrogenic } \\
\text { neurological } \\
\text { symptoms }\end{array}$ & $\begin{array}{c}\text { Ectopic } \\
\text { ossification }\end{array}$ & $\begin{array}{c}\text { Traumatic } \\
\text { arthritis }\end{array}$ \\
\hline 3D model group & 48 & 1 & 1 & 1 & 2 \\
\hline Routine operation group & 48 & 6 & 5 & 3 & 4 \\
\hline$\chi^{2}$ test & & & 9.66 & & \\
\hline P-value & & & 0.002 & & \\
\hline
\end{tabular}

could clearly display the acetabular fracture morphology in patients (Fig. 1B), thus helping observe the fracture type and design the operative plan. The reduction of acetabular fracture in patients was good at 3 days after operation (Fig. 1C). CT scan at 6 months after operation showed that the acetabular morphology was well restored (Fig. 1D).

\section{Discussion}

The acetabulum is in a deep concave hemispherical shape with a radius of $\sim 1.8 \mathrm{~cm}$, which is composed of anterior column and posterior column intersecting and overarching 60 degrees. There are abundant muscles, nerve networks, blood vessels and anastomotic branches around the acetabulum, the physiological structure is complex, and more bleeding and porosis will be easily caused after injury (12). With the rapid social development, increasingly more patients suffer from comminuted and irregular acetabular fractures due to high-energy impact (traffic accidents, falling objects and heavy impact). At the same time, fractures in other sites or organ injuries in the thoracic cavity, abdominal cavity and pelvic cavity are concurrent, seriously affecting the quality of life of patients and increasing the social burden. Currently, the acetabular fracture is divided into simple fracture and complex fracture according to the principle of Letournel E. The simple fracture includes fractures in the anterior wall, anterior column, posterior wall, posterior column and transverse site, while the complex fracture includes posterior column fracture accompanied with posterior wall 
Table V. Comparison of excellent-good rates of reduction quality of acetabular fracture and hip joint function after operation between the two groups (\%).

\begin{tabular}{lccc} 
Groups & $\mathrm{n}$ & $\begin{array}{c}\text { Reduction quality of } \\
\text { acetabular fracture }\end{array}$ & Hip joint function \\
\hline Routine operation group & 48 & $77.08(17 / 20)$ & $83.33(19 / 21)$ \\
3D model group & 48 & $81.25(20 / 19)$ & $87.5(24 / 18)$ \\
t-test & & 0.25 & 0.33 \\
P-value & & 0.61 & 0.56 \\
\hline
\end{tabular}

77.08 and 81.25 represent the excellent and good rates of postoperative patients. (17/20) represents that 17 patients had excellent postoperative joint recovery, 20 patients had good recovery, and 11 cases had poor recovery.

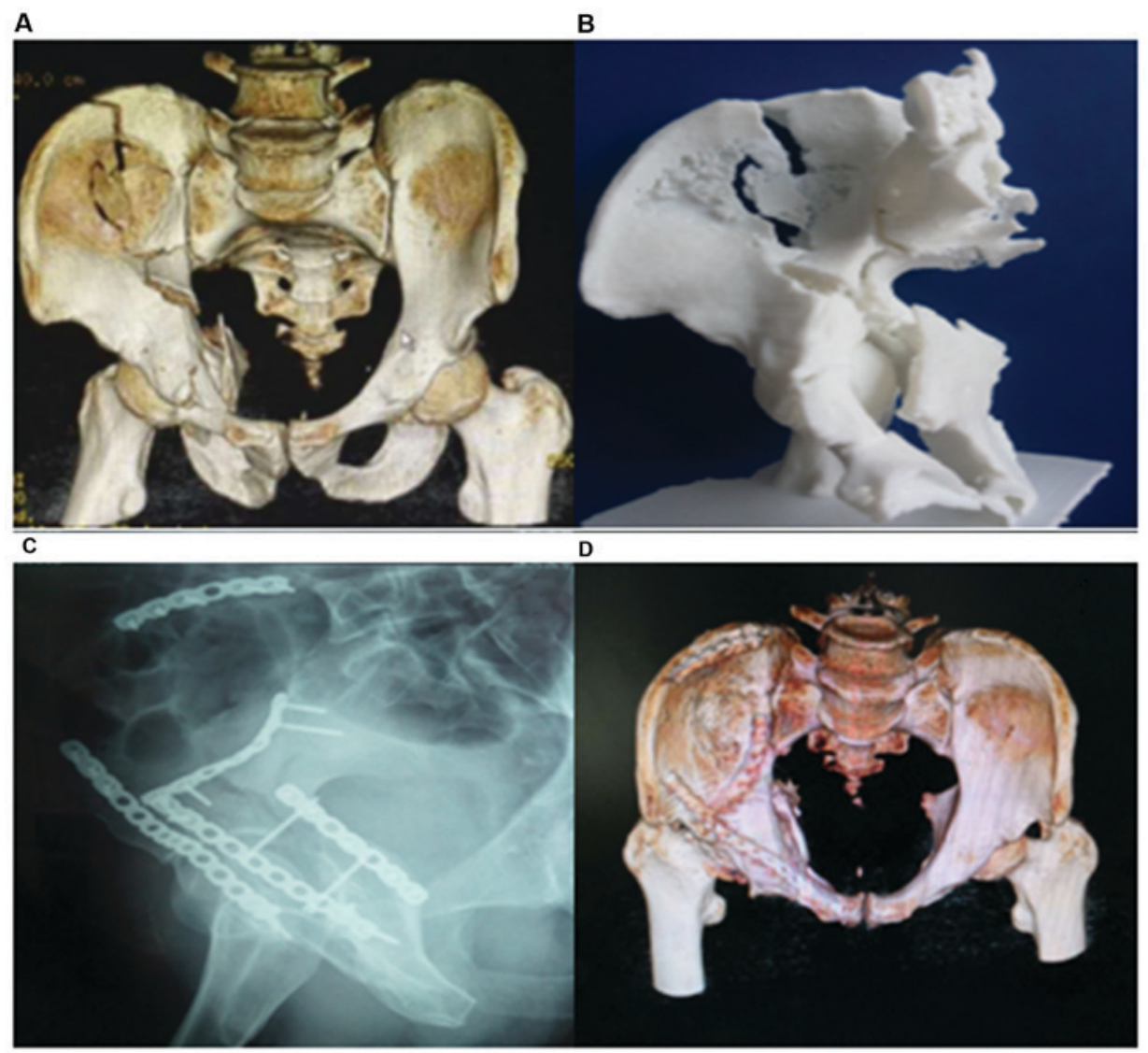

Figure 1. A 45-year-old male patient suffering from the right acetabular fracture complicated with craniocerebral and chest injuries due to a car accident. (A) Preoperative orthotopic CT scan shows both-column fracture; (B) preoperative 3D printing model; (C) X-ray fluoroscopy at 3 days after operation; and (D) CT scan at 6 months after operation.

fracture, transverse fracture accompanied with posterior wall fracture, anterior column or anterior wall fracture accompanied with posterior column or transverse fracture, T-shaped fracture and both-column fracture. Operative treatment is a preferred choice for clinicians, which, in particular, can better realize the anatomical reduction and fixation, and recover the function early for complex fracture with dislocation. However, operative treatment of acetabular fracture is also faced with many difficulties, such as the complex anatomical structure, selection of surgical approach, complex reduction and severe postoperative complications. How to improve the quality of operation, shorten the treatment time, reduce the incidence rate of postoperative complications and restore the physiological function of patients is the key to the treatment of patients with acetabular fracture.

With the development of X-ray, CT technique and so on, clinicians can conduct the simple 2D image analysis for the complex and comminuted fractures, but the fracture morphology cannot be reflected intuitively. According to Schreiner et al (13), clinicians are limited by factors such as the angle of the fracture site or overlapping fracture patches and occlusion, which often leads to long operation time, excessive blood loss and uneven joint surface, resulting in high incidence of postoperative complications. Therefore, the 
simple 2D X-ray examination and CT scan fail to meet the clinical needs of orthopedics.

The acetabular fracture is mostly an articular surface fracture, and the local bone shape is irregular, so multi-angle and all-round observation is needed before the operative plan is developed. Computer virtual technique overcomes the shortcomings of 2D images, such as the lack of stereoscopic effect and overlapping among tissues. Computer simulation reduction technique can reconstruct the stereoscopic and intuitional 3D model of the acetabular fracture through X-ray examination and CT scan, and observe from any angle through such functions as translation and rotation (14), which can help clinicians, especially those with inadequate clinical experience, understand the disease more comprehensively and develop therapeutic regimen. Computer virtual technique provides the virtual therapeutic environment for clinicians and simulates the clinical operation steps, and site and angle of internal fixation, which, compared with conventional operation, is characterized by intuition, no damage, visualization and repeatability (15). Moreover, computer virtual technique can develop an individualized therapeutic regimen based on the condition of patients, and improve the clinical effect and postoperative life of patients, which has great value of social generalization.

As one of the important reforms in the medical field, $3 \mathrm{D}$ printing technique is a bridge between computer virtual technique and realistic clinical operation. The 3D physical model displaying the acetabular fracture of patients perfectly can clearly identify the fracture line, broken bone fragment and its positional relation. In particular, the steel plate and screw can be selected for the quadrilateral and acetabular reduction and fixation according to the 3D physical model of acetabular fracture, followed by pre-bending treatment before operation, thus effectively reducing the operation time, improving the safety and optimizing the operative plan (16).

Results of this study revealed that the operation time, amount of intraoperative bleeding and times of intraoperative fluoroscopy in 3D model group were significantly reduced, and differences were statistically significant compared with routine operation group $(\mathrm{P}<0.05)$. The incidence rate of postoperative complications in the 3D model group had a statistically significant difference compared with that in the routine operation group $(\mathrm{P}<0.05)$. Besides, no statistically significant differences were found in the Matta imaging score at 3 days after operation and Harris score at 6 months after operation between the two groups $(\mathrm{P}>0.05)$. The above results indicate that computer virtual reduction combined with the $3 \mathrm{D}$ printing technique can obviously reduce the operation time, amount of intraoperative bleeding and incidence rate of postoperative complications of patients without adverse effects on the postoperative acetabular healing and function of patients. It can reduce the error and the surgical injury during the operation. There was no adverse effect on postoperative recovery of joint function. The main reason is that computer virtual reduction combined with the $3 \mathrm{D}$ printing technique can accurately restore the operative plan designed before operation and improve the accuracy and safety of operation, and the operation is simple and less time-consuming, making up for defects of the existing method of screw implantation. However, there are also some shortcomings in computer virtual reduction combined with the 3D printing technique. Firstly, high-quality CT images are required for the operation to improve the accuracy of model and avoid the operative error. Lou et al (17) considered that $3 \mathrm{D}$ objects can improve the clinical practice ability to a certain extent, but this technology cannot reflect the soft tissue, blood vessels, nerves and other conditions of the bone injury site. In the process of operation, the guidance of experienced clinicians is needed. Secondly, it is the skeletal structure that is scanned and printed in the operation, excluding the soft tissues, blood vessels and nerves, around the bone. Therefore, the operative plan design differs from the actual plan implemented during operation, and experienced clinicians are needed for guidance. Thirdly, the 3D printing of pelvis takes a long time, so it is not applicable for emergency patients. Therefore, clinician cannot rely completely on the operative plan designed in advance during operation, but should provide the corresponding treatment based on the actual condition of patients. There are some limitations in this study. Due to the short study time, the number of cases included in this study is not that large. It can prove the advantage of the computer virtual restoration in combination with the $3 \mathrm{D}$ printing to a certain degree, but its application effect remains to be tested by time and patients, which will be the focus of our future research. We will continue to add clinical cases and long-term follow-ups to provide guidance for the development of computer virtual reset combined with the $3 \mathrm{D}$ printing technology in clinic.

In conclusion, computer virtual technique can provide the individualized operative plan for patients, and 3D printing technique creates a bridge between virtual technique and realistic operation. Their combination can remarkably improve the safety and accuracy of clinical operation, which has a higher clinical application value and great social significance.

\section{Acknowledgements}

Not applicable.

\section{Funding}

No funding was received.

\section{Availability of data and materials}

The datasets used and/or analyzed during the present study are available from the corresponding author on reasonable request.

\section{Authors' contributions}

LW and $\mathrm{XZ}$ were involved in the conception and design of the study. KL and PC drafted the manuscript. LW, XZ and $\mathrm{SZ}$ recorded and analyzed the basic data of patients. $\mathrm{KL}, \mathrm{PC}$ and JL were responsible for CT scan analysis. LW and GW were in charge of surgical approach and statistical analysis. The final version was read and approved by all the authors.

\section{Ethics approval and consent to participate}

The study was approved by the Ethics Committee of The Second Affiliated Hospital of Luohe Medical College (Luohe, 
China). Signed informed consents were obtained from the patients and/or guardians.

\section{Patient consent for publication}

Not applicable.

\section{Competing interests}

The authors declare that they have no competing interests.

\section{References}

1. Keil H, Beisemann N, Schnetzke M, Vetter SY, Swartman B, Grützner PA and Franke J: Intraoperative assessment of reduction and implant placement in acetabular fractures-limitations of 3D-imaging compared to computed tomography. J Orthop Surg Res 13: 78, 2018.

2. Ma L, Zhou Y, Zhu Y, Lin Z, Chen L, Zhang Y, Xia H and Mao C: 3D printed personalized titanium plates improve clinical outcome in microwave ablation of bone tumors around the knee. Sci Rep 7: 7626, 2017.

3. Liu X, Zeng CJ, Lu JS, Lin XC, Huang HJ, Tan XY, Cai DZ and Orthopedics DO: Application of 3D printing and computer-assisted surgical simulation in preoperative planning for acetabular fracture. Nan Fang Yi Ke Da Xue Xue Bao 37 378-382, 2017 (In Chinese).

4. Judet R, Judet J and Letournel E: Fractures of the acetabulum: classification and surgical approaches for open reduction. Preliminary report. J Bone Joint Surg Am 46: 1615-1646, 1964.

5. Uei H, Tokuhashi Y, Maseda M, Nakahashi M, Sawada H, Matsumoto K and Miyakata H: Exploratory analysis of predictors of revision surgery for proximal junctional kyphosis or additional postoperative vertebral fracture following adult spinal deformity surgery in elderly patients: A retrospective cohort study. J Orthop Surg Res 13: 252, 2018

6. Deng C, Ni WD, Guo SQ, Luo G, Shui W and Qiao B: Operative treatment of delayed acetabular fractures through combined anterior and Kocher-Langenbeck approaches. Zhonghua Wai Ke Za Zhi 56: 196-200, 2018 (In Chinese).
7. Clough TM, Alvi F and Majeed H: Total ankle arthroplasty: What are the risks? Bone Joint J 100-B: 1352-1358, 2018.

8. Cao D, Zhang S, Yang F, Shen K and Tan Z: Hidden blood loss and its influencing factors after percutaneous kyphoplasty surgery: A retrospective study. Medicine (Baltimore) 97: e0435, 2018.

9. de Vries LM and Neve WC and Steens J: Prosthesis retention after an infected hip prosthesis: Hip fractures versus primary total hip prosthesis, data from 1998-2015. J Bone Jt Infect 3: 118-122, 2018.

10. Bücking TM, Hill ER, Robertson JL, Maneas E, Plumb AA and Nikitichev DI: From medical imaging data to 3D printed anatomical models. PLoS One 12: e0178540, 2017.

11. Ho D, Squelch A and Sun Z: Modelling of aortic aneurysm and aortic dissection through 3D printing. J Med Radiat Sci 64: 10-17, 2017.

12. Porter SE, Russell GV, Dews RC, Qin Z, Woodall J Jr and Graves ML: Complications of acetabular fracture surgery in morbidly obese patients. J Orthop Trauma 22: 589-594, 2008

13. Schreiner AJ, Schmidutz F, Ateschrang A, Ihle C, Stöckle U, Ochs BG and Gonser C: Periprosthetic tibial fractures in total knee arthroplasty - an outcome analysis of a challenging and underreported surgical issue. BMC Musculoskelet Disord 19: 323, 2018.

14. Maini L, Verma T, Sharma A, Sharma A, Mishra A and Jha S: Evaluation of accuracy of virtual surgical planning for patient-specific pre-contoured plate in acetabular fracture fixation. Arch Orthop Trauma Surg 138: 495-504, 2018.

15. Wang YC, Ma Y, Yu WZ, Li YF and Liu YH: Application of the computer-assisted virtual reduction combined with 3D printing technique in acetabular fractures. Zhongguo Gu Shang 30: 627-632, 2017 (In Chinese).

16. Arabnejad S, Johnston B and Tanzer M and Pasini D: Fully porous 3D printed titanium femoral stem to reduce stress-shielding following total hip arthroplasty. J Orthop Res 35: 1774-1783, 2017.

17. Lou Y, Cai L, Wang C, Tang Q, Pan T, Guo X and Wang J: Comparison of traditional surgery and surgery assisted by three dimensional printing technology in the treatment of tibial plateau fractures. Int Orthop 41: 1875-1880, 2017. 